\title{
Features and Capabilities of the Digital Art Community
}

\author{
Olga Perova \\ Artist, Digital painter, Master of Engineering and Technology
}

\begin{abstract}
The online community of digital artists includes several tens of millions of people - registered members and active users of the largest online galleries of digital art and social networks for artists. The opportunity to publish their work on the Internet is often for emerging artists the only way to get the attention of the audience and gain their fans. An amateur artist is able to bring his work to a professional level, to turn it into a main source of income - by selling the rights to his work, producing products based on it, or by financially supporting active users of the community.

Unfortunately, publicity is fraught with certain risks. For example, sharply negative and offensive comments and harassment in social networks. Unfriendly reception in the community can be a cause for the artist to forever engage with creativity. Criticism of a work of art is not always objective and justified; Often it is a catalyst for scandals, with accusations of racism, sexism and so on.

With all the advantages and disadvantages, it should be noted that the online art community is a force capable of influencing the modern entertainment industry.
\end{abstract}

Keywords: online community, digital painting, gallery, visual arts

The key factors that influenced the formation of the existing online community of digital artists and art fans were the spread of the worldwide Internet and the availability of personal computers with professional software (graphic editors).

In 1991, the World Wide Web became publicly available on the Internet; in 2019, the number of Internet users is 4.388 billion [1]. In parallel with the growth of the network, graphic editors such as Adobe Photoshop were developed and improved. Its first version appeared in 1987; To date, version 20 is available to users.

The number of registered users of the largest online communities for artists amounts to tens of millions of people. For example: DevaintArt - 44 million people; Pixiv - more than 20 million people; Behance - more than 10 million people. The number of active users of social Internet service and photo hosting Pinterest, which allows you to add images online, is approaching 300 million people per month. Finally, Instagram is an application for sharing photos and videos with elements of a social network, for 2018 there were 1.1 billion registered users, which is about $15 \%$ of the population of the Earth. Most of the Instagram 
content is made up of photos and videos, however, many professional digital artists and digital creativity lovers also have Instagram accounts and regularly publish their work there.

Online communities allow creative people to publish their work, regardless of the professional level of the artist. This is what distinguishes them from traditional art galleries, where it is quite difficult for a beginner to demonstrate his work. The possibility of publishing in an online gallery can have both positive and negative effects.

In the first case, the artist collects positive comments and gets his first audience. The presence of a positive audience response has a tremendous motivational effect. In addition to the incentive to further work, the artist can more accurately determine the tastes and needs of the audience, in order to then commercially orient his work. For greater efficiency, it makes sense to engage in a kind of self-promotion, that is, to share your work in all available social networks and creative communities.

In the second case (negative effect), the artist runs the risk of facing a negative in relation to his art. Unfortunately, "toxicity" on the Internet has become a real problem; bullying and harassment in the comments lead to the saddest consequences. A few derogatory harsh words are able to forever discourage the desire to continue to engage in creativity.

Developing the topic of publishing artwork in online galleries, you need to mention this kind of creativity as "fan art". Fan art is a derivative work based on any original work. As a rule, the artist draws a character from a video game, film, cartoon, and so on. There are no detailed studies and reports on the percentage of fan-art in the work of digital artists, but just look at the DeviantArt Gallery's "Popular 24 Hours" collection to find out that the share of fan-art among the works is at least $50 \%$ of the total numbers [2].

Formed a scheme in which the artist is obliged to closely monitor current trends in popular culture and draw all the emerging characters as quickly as possible, until they passed their popularity. The resulting fan-art is published in online galleries; the community, which the new character has not yet bored, favorably accepts fan-art; the artist gets a new audience; character gets extra advertising and gaining popularity.

Most often, the described scheme applies to video games. For example, when the company "Nintendo" adds a new character - a pokemon (a "pokemon" - a pocket monster, a magical creature) to one of its "Pokemon" games, then literally in a few hours the first 
pictures with this character begin to appear on the network. As long as this character is interesting to fans of Pokemon games - fan art with its participation will be popular.

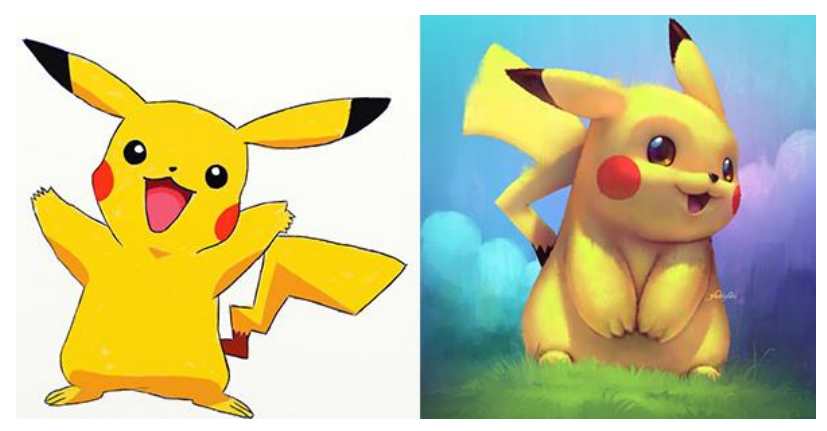

Fig.1. On the left is the original pokemon; right - fan art by Cássio Yoshiyaki

In other words, the artist must always be alert and quickly draw all the newest. Of course, this statement is true only for fan-art and does not concern those who draw original works.

But both fan art and original artwork published in online communities are not the end point. They, in turn, give impetus to the production of goods (figures, souvenirs, printed materials) or are embodied in the costumes of cosplayers ("cosplay" is a popular hobby, which consists in dressing up computer games, cinema, etc. characters) in costumes.

It is obvious that the audience of online communities does not consist only of fans and amateur artists. Representatives of the entertainment industry and manufacturers of goods are in search of good and sought-after ideas, new talents. The most popular illustrations can bring to their creator a one-time or multiple income by selling copyrights or deducting interest from the sale of goods created on the basis of this illustration.

Returning to the issue of criticism in the community, it should be noted that in exceptional cases the community opinion becomes an influential force. A good example is shown by the story of a film based on the Sonic the Hedgehog video game series, the rights to create and distribute which in 2013 were acquired by Sony Pictures Entertainment [3]. 
In 2018, the trailer for the film came out, but most of Sonic's fans were unhappy with his image: face and proportions were the most criticized. The illustrator LadyGT has published a revised version of Sonic on social networks.

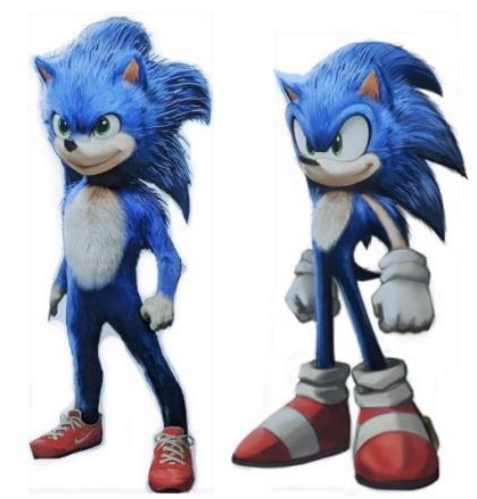

Fig.2. On the left is Sonic the hedgehog proposed by the filmmakers; on the right an option from the artist LadyGT

Then similar offers from other digital artists and designers began to appear. Probably, the criticism would not have been heard, if the artists had not supported their opinion with images of the "correct" Sonic. As a result, the film studio decided to remake the character design according to the wishes of the fans.

But criticism is not always constructive. Multi-million community can not be unanimous on all issues. Periodically, the work of artists provoke scandals on objectification, racism and others. So, in 2018, digital artist working under the nickname Evulchibi, published a fan-art on the superhero "Black Panther". However, the artist portrayed the hero as white, while the original character is a black African.

This work provoked a wave of angry comments; for several days, the author was accused of racism, colonization, hypocrisy and the infringement of the rights of black people. The discussion ended when it turned out that the artist himself was black [4].

From all this, it follows that the online art community is heterogeneous. But its undoubted advantage in the opening opportunities:

- to help novice artists to gain recognition and popularity;

- directly or indirectly influence the entertainment industry and its products; 
- to be a place of communication for connoisseurs of modern art in general, and digital painting in particular.

\section{References}

1. Global Digital 2019 reports URL: https://wearesocial.com/blog/2019/01/digital-2019global-internet-use-accelerates

2. DeviantArt URL: https://www.deviantart.com/

3. Live-Action Sonic the Hedgehog Movie Is Speeding into Development URL: https://www.slashfilm.com/sonic-the-hedgehog-live-action-movie/

4. Medialeaks [In Russian] URL: https://medialeaks.ru/0203xsh-fan-art-of-black-panther/ 5. Perova O. Digital painting: the use of computer graphics in art // International journal of applied and fundamental research. - 2019. - № 1; URL: http://www.sciencesd.com/479-25504 (29.01.2019). 